\title{
Demografia Médica em Portugal: Análise Prospetiva
}

\author{
Demography of Physicians in Portugal: Prospective Analysis
}

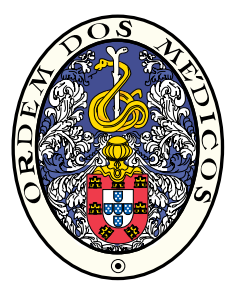

\author{
Paula SANTANA ${ }^{1}$, Helena PEIXOTO ${ }^{2}$, Nuno DUARTE ${ }^{2}$ \\ Acta Med Port 2014 Mar-Apr;27(2):246-251
}

\section{RESUMO}

Introdução: Partindo da caracterização demográfica dos médicos em Portugal e através do desenvolvimento de diferentes cenários prospetivos, modelou-se a evolução previsível da dotação de médicos, por especialidade, no horizonte de 2025.

Material e Métodos: Foi desenvolvido um Modelo da Oferta, que representa a capacidade de formação de médicos instalada no sistema, e um Modelo de Necessidades, que perspetiva futuros possíveis para a dotação de profissionais.

Resultados: Cada um dos modelos incluiu diferentes cenários, de acordo com variáveis de contexto diversas (introdução ou não de limites à capacidade formativa pós-graduada, evolução demográfica, rácios recomendados pelos Colégios de Especialidade...).

Discussão/Conclusão: Da confrontação dos modelos desenvolvidos e dos respetivos cenários resultou a conclusão de que o sistema tem capacidade para suprir as necessidades de médicos no horizonte de 2025 em todos os cenários modelados, gerando mesmo profissionais que poderão não ser absorvidos pelo sistema de saúde.

Palavras-chave: Demografia; Portugal; Médicos / provisão \& distribuição.

\section{ABSTRACT}

Introduction: The study modelled the physicians predictable allocation by medical specialty, in the horizon of 2025. It was based on the physicians demographics in Portugal and through the development of different future scenarios.

Material and Methods: In the study was developed a Supply Model, which represents the capacity of medical training installed in Portugal, and a Necessity Model that perspective possible futures for the provision of medical professionals.

Results: Each model comprehends several scenarios, according to different context variables (introduction or not of limits to postgraduate training capacity, demographic trends, ratios recommended by Colleges Specialty ...).

Discussion/Conclusion: The models comparison lead to the conclusion that the training system has the capacity to meet the needs of physicians in all scenarios developed, and it even generates a surplus of medical professionals that may not be absorbed by the healthcare system, in the horizon of 2025.

Keywords: Demography; Physicians / supply \& distribution; Portugal.

\section{INTRODUÇÃO}

Num sistema de elevada complexidade e que exige recursos altamente qualificados como é o sector da saúde, o conhecimento dos recursos humanos afetos ao seu funcionamento é fundamental. ${ }^{1}$ Constitui uma questão extremamente complexa, ${ }^{2}$ com múltiplos aspetos e interações que cobrem vertentes muito diversas e que determinam a eficácia e a eficiência do sistema de saúde ${ }^{3,4}$ sendo, simultaneamente, determinados por estas. ${ }^{5}$ Importa, pois, refletir sobre o futuro dos recursos humanos no setor da saúde, analisar tendências de evolução no contexto envolvente e perspetivar necessidades no futuro próximo, ${ }^{2,6,7}$ incluindo a formação pré e pós-graduada. ${ }^{8}$ Neste contexto, o Ministério da Saúde promoveu Estudos em 2007 e 20099,10 que, além de caracterizarem os recursos humanos do Serviço Nacional de Saúde (nomeadamente os médicos), desenvolveram cenários de projeção da respetiva evolução.

Num contexto de profundas alterações vivenciadas pelo sistema de saúde em Portugal, nomeadamente ao nível da dotação de recursos humanos do sector, foi considerado essencial proceder à atualização da caracterização dos médicos no Sistema de Saúde nacional, bem como dos respetivos cenários prospetivos de evolução. ${ }^{11}$ No atual panorama vivenciado no sector da saúde, é também importante alargar esta análise a todos os clínicos ativos no sistema, ou seja, considerar não exclusivamente os médicos do Serviço Nacional de Saúde, mas também os afetos aos sectores privado e social; em suma, considerar na análise a efetuar, todos os profissionais do Sistema Nacional de Saúde.

Por outro lado, as mudanças de contextos organizacionais e as reformas no sector da saúde, assim como as profundas alterações verificadas na situação profissional de muitos médicos, com a existência de uma vaga de aposentações no sistema público (em grande parte antecipadas relativamente à idade mínima de reforma dos profissionais em causa), determinaram a necessidade de desenvolver uma base de conhecimento que permitisse: i) efetuar um adequado planeamento de profissionais de medicina, nomeadamente ao nível da previsão de necessidades de formação (inicial e especializada) e ii) promover a adequação do número de profissionais (tanto a nível global como por especialidade médica) às necessidades de funcionamento do Sistema de Saúde.

O presente artigo decorre de um estudo elaborado para

1. Centro de Estudos em Geografia e Ordenamento do Território. Grupo de Investigação em Geografia da Saúde. Universidade de Coimbra, Coimbra. Portugal.

2. Grupo de Investigação em Geografia da Saúde. Universidade de Coimbra. Coimbra. Portugal.

Recebido: 17 de Janeiro de 2014 - Aceite: 25 de Fevereiro de 2014 | Copyright $\odot$ Ordem dos Médicos 2014 
a Ordem dos Médicos ${ }^{11}$ sobre a evolução prospetiva de médicos em Portugal que visava dois objetivos essenciais: i) a caracterização dos médicos habilitados a exercer medicina em Portugal; ii) a estimativa do número de médicos adequado à satisfação das necessidades de saúde da população no horizonte temporal de 2025.

A análise pretendeu enquadrar-se no contexto das reformas da saúde em curso, numa perspetiva de médio e longo prazo (horizonte temporal de 2025), visando identificar o gap entre as necessidades de cobertura destes profissionais (por especialidade) e o número expectável dos mesmos.

O Estudo incidiu sobre a distribuição dos profissionais pelas diferentes especialidades médicas e procura fornecer evidência para responder à questão recorrentemente formulada em Portugal e noutros países desenvolvidos ${ }^{6}-0$ número de médicos existente e em formação é o adequado às necessidades presentes e futuras do país?

\section{MATERIAL E MÉTODOS}

O estudo baseou-se em dados demográficos (população residente, e previsões da população para 2025, segundo o Instituto Nacional de Estatística) e em informação proveniente de duas fontes que, em conjunto, permitiram caracterizar o efetivo de médicos existente em Portugal em Dezembro de 2011: i) base de dados fornecida pela Administração Central do Sistema de Saúde contendo informação sobre os médicos afetos ao Serviço Nacional de Saúde no Continente; ii) base de dados fornecida pela Ordem dos Médicos respeitante aos profissionais inscritos na Ordem dos Médicos e que não possuíam vínculo ao Serviço Nacional de Saúde.

A partir da determinação e caracterização dos profissionais existentes em 2011 (número de profissionais por especialidade, idade, e.g.) procedeu-se à modelação de cenários de evolução (até 2025), perspetivando diferentes opções possíveis para a afetação de médicos ao funcionamento do Sistema de Saúde em Portugal.

A análise dos principais modelos teóricos de projeção de recursos humanos em saúde (baseados na oferta, na procura, na necessidade e em "benchmarking") demonstra que qualquer deles, isolado e per se, comporta vantagens e inconvenientes. No âmbito do estudo foi utilizado um modelo composto, de forma a minimizar as respetivas limitações e maximizar as suas potencialidades, tendo por base uma metodologia de projeção assente num macro-modelo sistémico, envolvendo a utilização integrada dos modelos de projeção da oferta e das necessidades, sobre uma evolução dinâmica das variáveis de suporte.

A opção pelo desenvolvimento de um modelo integrado de oferta e necessidades de profissionais médicos ancora-se na pesquisa levada a cabo tanto relativamente aos desenvolvimentos teóricos produzidos sobre a matéria da modelação prospetiva de necessidades de recursos humanos em saúde como, sobretudo, aos exercícios práticos de aplicação destes modelos efetuados em diversos países europeus e nos EUA. ${ }^{12}$ Foram ainda analisados em profun- didade os trabalhos relativos a Espanha, ${ }^{13}$ à Bélgica ${ }^{14}$ e aos Estados Unidos da América. ${ }^{15}$

Da análise levada a cabo resulta a conclusão de que parte dos modelos se baseia apenas nas tendências demográficas para avaliar a evolução da oferta e das necessidades de médicos, enquanto outros incorporam já outros elementos que visam aumentar a sua precisão, de que são exemplo, a nível da oferta, as tendências previstas de aposentação dos profissionais ou as suas taxas de mortalidade; ao nível da procura existem já modelos que procuram integrar nas suas componentes fatores como a evolução da despesa em saúde ou a previsão das tendências de evolução da skill-mix ao nível das profissões do sector da saúde. A introdução destes elementos representa uma sofisticação crescente na capacidade de previsão dos modelos, mas apresenta limitações decorrentes, entre outros fatores, da existência e da fiabilidade das fontes de informação a nível nacional.

Assim, partindo da dotação de médicos em 2011 e considerando os fluxos de entrada no sistema de saúde (através da formação pré e pós-graduada) e os fluxos de saída do sistema (por limite de idade, que se convencionou situar nos 70 anos), foram desenvolvidos dois modelos de base para estimar a evolução dos médicos em Portugal até 2025 , que integram diferentes cenários, de acordo com variáveis de contexto diversas - existência de limites para a capacidade formativa pós-graduada, evolução demográfica prevista para a população em Portugal, rácios de médicos por especialidade recomendados pelos Colégios de Especialidade da Ordem dos Médicos.

Nesta perspetiva, os modelos e respetivos cenários desenvolvidos foram os seguintes:

- Um modelo que se baseia na projeção da evolução das condições atualmente existentes até 2025, designado Modelo de Oferta. Este Modelo representa a capacidade instalada de produção de médicos no sistema de saúde e incorpora dois cenários, tendo como variáveis a capacidade formativa pós-graduada. Um cenário - Cenário Sem Limitações da Oferta Formativa -, assume que o sistema de internatos médicos tem capacidade para absorver, em cada ano, a totalidade dos diplomados em medicina que se candidatam à formação pós-graduada; outro cenário Cenário Com Limitações à Oferta Formativa -, baseia-se numa capacidade máxima de ingresso nos internatos de especialidade na ordem das 1550 vagas anuais (este limite corresponde à média entre os valores estimados pelo Ministério da Saúde para a capacidade formativa do Sistema de Saúde no futuro próximo). ${ }^{16}$

- Um Modelo de Necessidades, que considera a introdução de variáveis de contexto na definição de um efetivo de profissionais adequado às necessidades assistenciais do país. Este modelo incorpora também dois cenários diferenciados de evolução, cujas variáveis de base são as seguintes:

- Cenário de Manutenção dos rácios médico/população (por especialidade) existentes em 2011 e sua projeção para 2025; 
- Cenário Desejável que parte da definição dos rácios de cobertura médico/população considerados adequados em cada especialidade, com base nas indicações de 22 Colégios de Especialidade, e os adapta à evolução populacional prevista até 2025.

O Modelo da Oferta representa, assim, a capacidade nacional de produção de médicos e de especialistas, que se compara com o Modelo das Necessidades, que visa modelar o número de profissionais de que o País necessitará no horizonte temporal de 2025 , de acordo com os cenários concebidos. Do confronto dos dois modelos e dos respetivos cenários resulta a identificação dos desajustamentos entre a 'oferta' e a 'necessidade' de profissionais no horizonte temporal definido.

A realização do estudo deparou-se com algumas limitações metodológicas de que se destacam: i) a informação relativa aos profissionais inscritos na Ordem dos Médicos é limitada, não permitindo a sua afetação a sectores de atividade ou o cálculo da afetação do tempo de trabalho dos profissionais (ETI- Equivalentes a Tempo Integral), por exemplo; ii) a existência de 7500 médicos inscritos na Ordem dos Médicos que não têm especialidade conhecida, correspondendo a $17 \%$ dos profissionais; iii) o facto de as projeções com horizontes dilatados (2025) apresentarem um elevado grau de incerteza.

\section{RESULTADOS}

Em Dezembro de 2011 existiam 43247 médicos habilitados a exercer medicina em Portugal, dos quais $58 \%$ se encontravam afetos ao funcionamento do Serviço Nacional de Saúde no Continente.

Os médicos portugueses constituem um grupo profissional globalmente envelhecido, em que $54 \%$ dos seus membros tem mais de 50 anos, sendo que o escalão etário entre os 50 e os 59 anos concentra $30 \%$ de todos os profissionais. Em contrapartida, o escalão etário dos 40 aos
49 anos representa apenas $15 \%$ do total dos profissionais, correspondendo a um período de fortes restrições no acesso aos cursos de Medicina que se verificou nos anos 80 e 90.

A profissão médica é, ainda, uma profissão globalmente equilibrada em termos de género, com $51 \%$ de médicos do género feminino.

As últimas décadas evidenciam, porém, uma alteração no padrão demográfico dos médicos em Portugal, com um acentuado rejuvenescimento dos profissionais $(31 \%$ do total têm até 39 anos) e um significativo aumento da sua feminização - nos escalões etários até aos 39 anos as profissionais do género feminino são quase o dobro dos seus colegas do género masculino.

A larga maioria dos médicos habilitados ao exercício da medicina em Portugal é de nacionalidade portuguesa (mais de $90 \%)$.

Como referimos, a modelação de cenários teve em conta as entradas e as saídas previstas do sistema de saúde no horizonte temporal definido. Assim, no que se refere às entradas decorrentes do sistema de formação pré-graduada em Medicina, verifica-se um aumento muito significativo tanto do número de vagas para ingresso nos cursos (superior a $250 \%$ entre 1995 e 2010), como do número de diplomados, que quadruplicou entre 1996 e 2010 (neste último ano diplomaram-se em Medicina, em universidades portuguesas, 1280 alunos) (Fig. 1). Considerando uma lógica de continuidade e de não alteração das condições existentes no que se refere à capacidade formativa instalada, até 2025 diplomar-se-ão cerca de 27000 mestres em Medicina em Portugal, correspondendo a um 'débito' anual de cerca de 1900 diplomados a partir de 2016.

Quanto ao outro elemento que alimenta as entradas no sistema de saúde, a formação médica pós-graduada, verifica-se que entre 2006 e 2012 o Ministério da Saúde disponibilizou um número de vagas para o Internato Médico

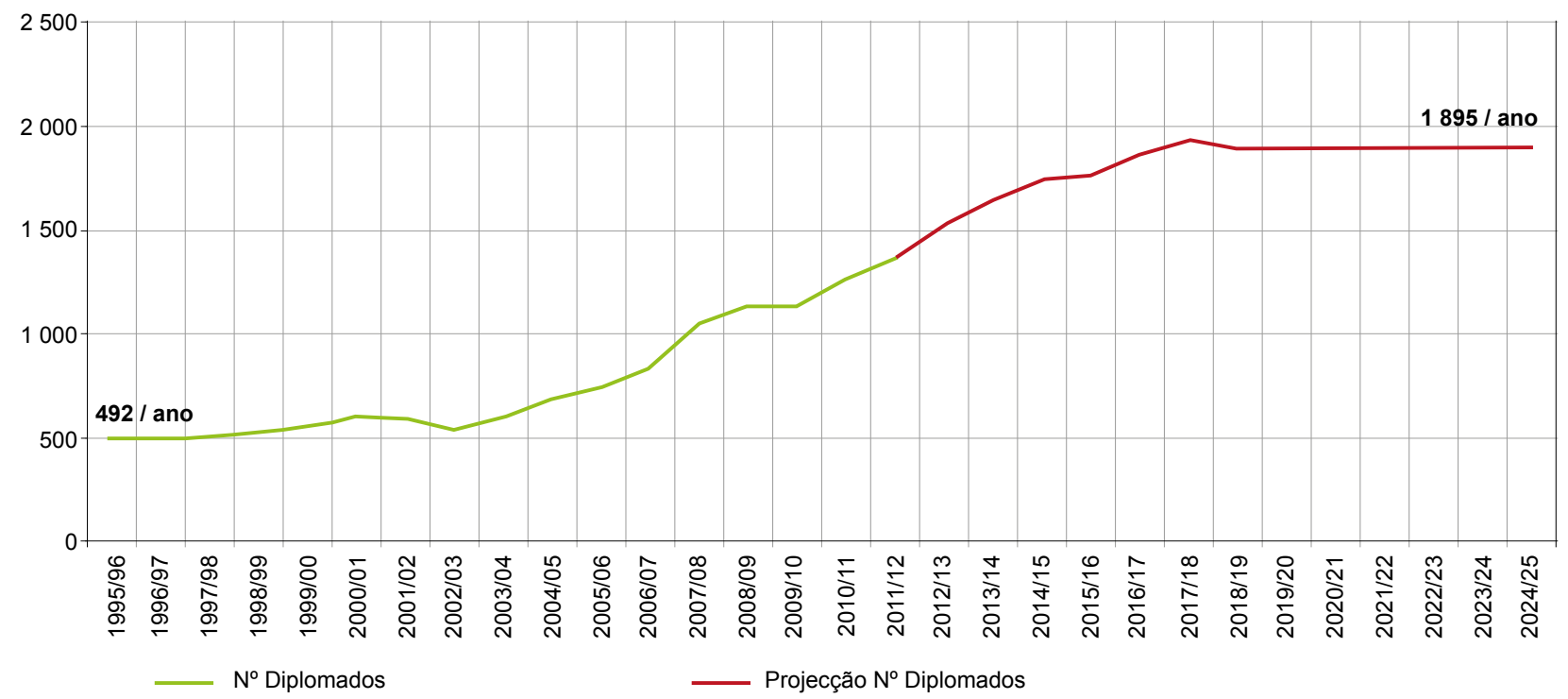

Figura 1 - Evolução do Número de Diplomados em Medicina (1996 - 2025) 


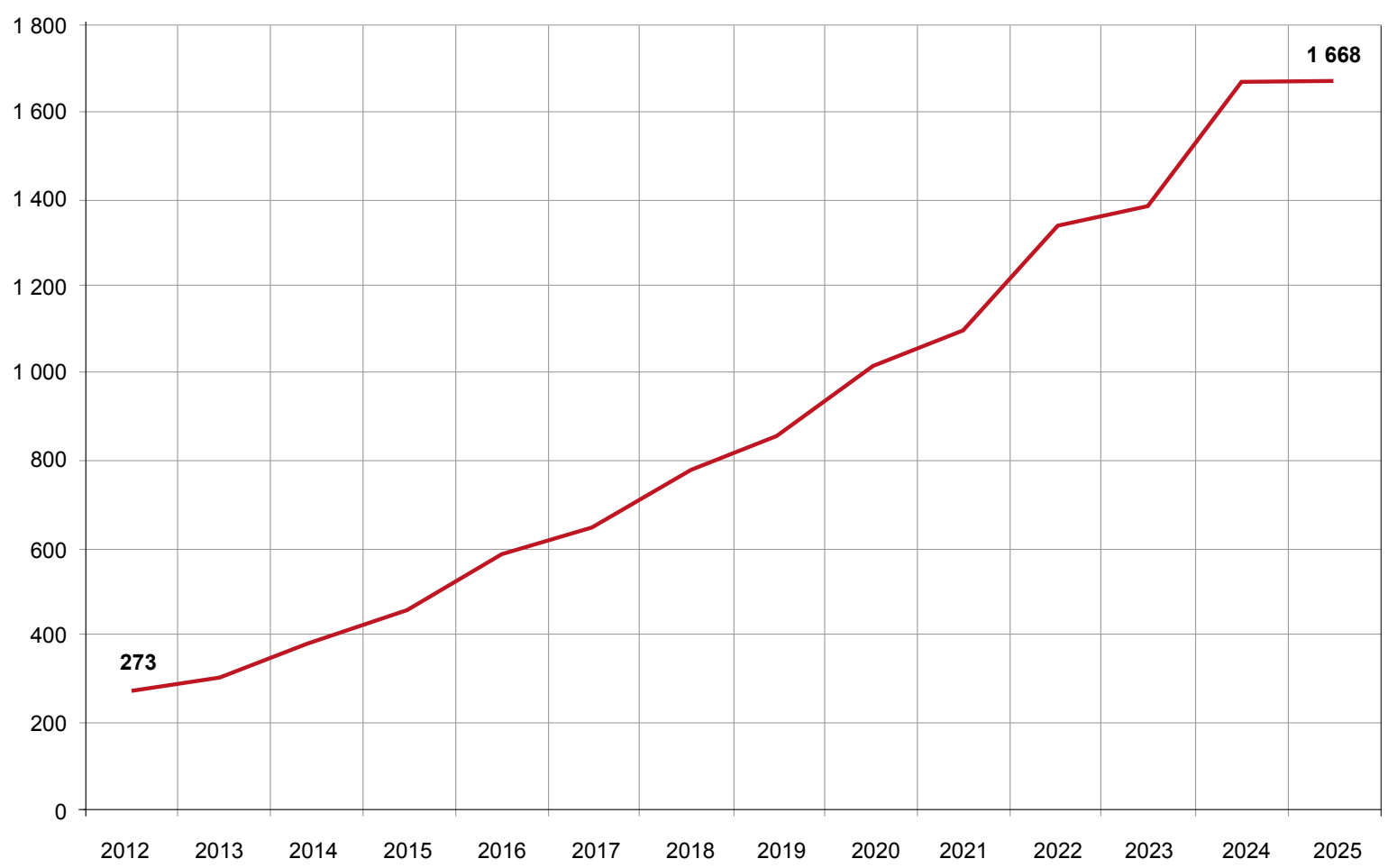

Figura 2 - Previsão de Abandono Anual da Atividade por parte dos Médicos no Sistema de Saúde por atingirem 70 anos (2012 - 2025)

adequado ao número de candidatos, tanto para a frequência do 'ano comum' como para ingresso na formação específica. Assim, verifica-se um aumento global do número de vagas para ingresso nos dois tipos de formação (inicial e específica), que no caso da formação específica atingiu os $67 \%$ entre 2006 e 2012, correspondendo a um aumento líquido de cerca de 600 vagas. No entanto, é previsível que a capacidade de formação do sistema se encontre esgotada, sendo provável que estabilize nas 1550 vagas, como referimos.

No que se refere às saídas do sistema de saúde previstas até 2025, que se convencionou estabelecer nos 70 anos, o contingente de profissionais que cumpre esta condição totalizará os 12473 ao longo do período de projeção, correspondendo a $32 \%$ dos profissionais em idade ativa em 2011 (Fig. 2).

As simulações efetuadas de acordo com os cenários desenvolvidos apresentam os seguintes resultados:

i) A capacidade de produção instalada no sistema de saúde (correspondente ao Modelo da Oferta) resulta num total de médicos no sistema, em 2025, quantificado entre os 51800 e os 51 900, de acordo com os diferentes cenários, incluindo médicos especialistas, médicos em formação e médicos sem qualquer especialidade. A concretização destes cenários traduz-se num aumento de cerca de $20 \%$ no total de médicos em Portugal.

ii) A capacidade de produção de médicos especialistas instalada no sistema de formação nacional, expressa no Modelo da Oferta, representa um aumento de especialistas no sistema de saúde, no horizonte de 2025 , entre os $32 \%$ no Cenário Sem Limitações à Capacidade Formativa no In- ternato Médico (correspondendo a 20795 novos especialistas) e os $21 \%$ no Cenário Com Limitações à Capacidade Formativa no Internato Médico (correspondendo a 17891 novos especialistas);

iii) As necessidades de especialistas a afetar ao sistema de saúde em 2025, expressas no Modelo das Necessidades, resultam numa variação relativamente a 2011 que se situa entre um aumento de $7 \%$ no Cenário Desejável (14 363 novos especialistas) e um decréscimo de $-2 \%$ no Cenário de Manutenção (11 913 novos especialistas) (Fig. 3).

Verifica-se, assim, um desajustamento entre a capacidade de produção/formação instalada e as necessidades estimadas através dos cenários desenvolvidos. A dimensão dos desajustamentos apurados varia entre os seguintes valores:

- No Cenário da Manutenção teríamos 8882 novos especialistas que não seriam absorvidos relativamente ao Cenário Sem Limitações e 5978 que seriam excedentários relativamente ao Cenário Com Limitações;

- No Cenário Desejável o excedente de novos especialistas não absorvidos situar-se-ia entre os 6432 relativamente ao Cenário Sem Limitações e os 3528 no Cenário Com Limitações.

\section{DISCUSSÃO}

O desenvolvimento deste tipo de exercícios prospetivos é fortemente condicionado pela fiabilidade da base informacional ${ }^{7}$ em que assentam, carecendo Portugal de um conhecimento mais aprofundado da atividade médica (exterior ao Serviço Nacional de Saúde) que se desenvolve no país. 


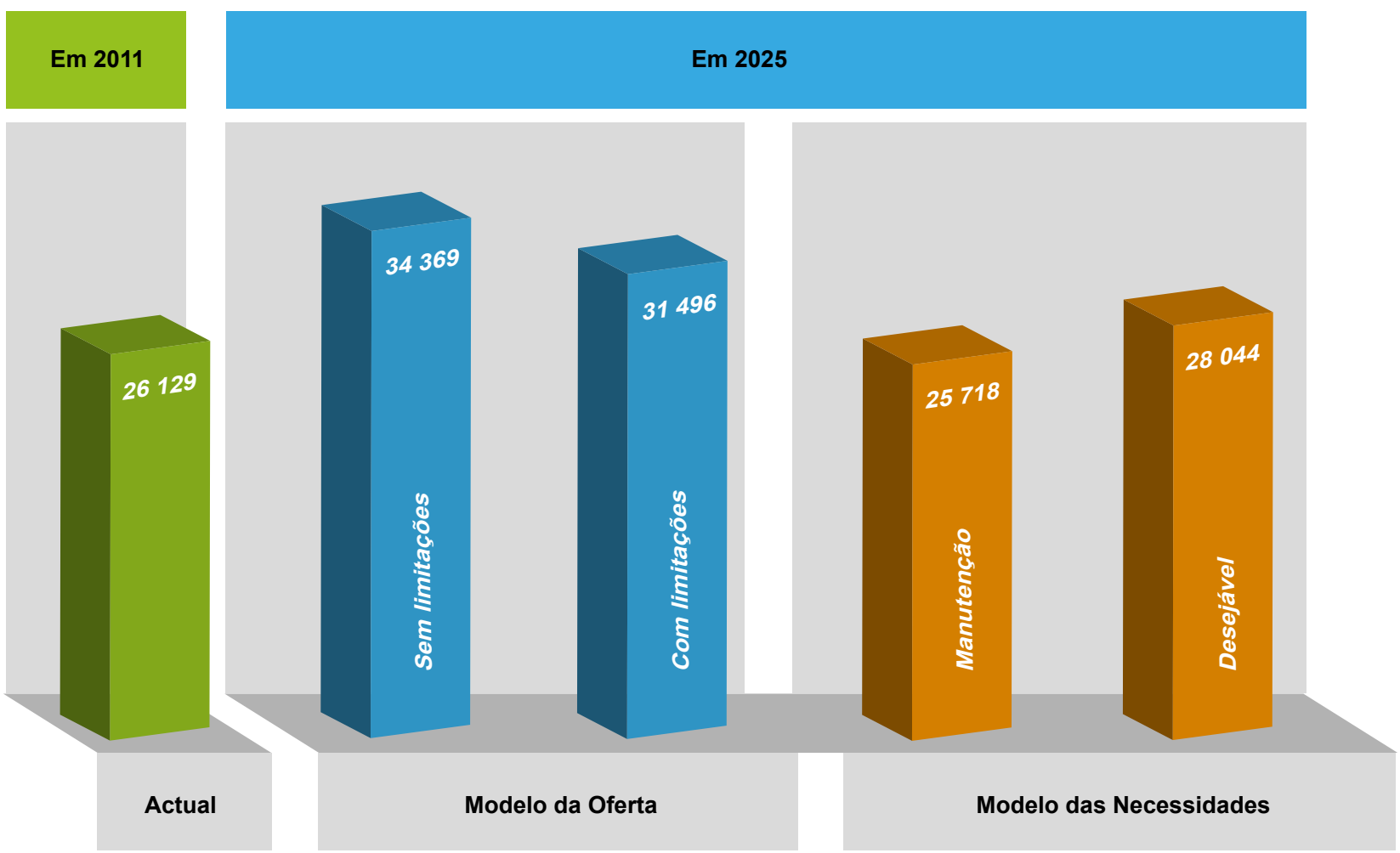

Figura 3 - Comparação entre Cenários - Médicos Especialistas

Adicionalmente, face à diversidade e complexidade dos fatores que intervêm na evolução da dotação de médicos num país, é aconselhável uma monitorização regular deste fenómeno, que permita a introdução de ajustamentos e correções de percurso. ${ }^{2}$

O Estudo que realizámos não considerou a distribuição geográfica dos médicos nem as restantes profissões do sector da saúde, que constituem fatores importantes para evolução futura da profissão médica. A estimativa de quantitativos de profissionais a nível nacional poderá revelar-se insuficiente para suprir necessidades em áreas geográficas mais excêntricas ou menos atrativas, enquanto a evolução de outras profissões como a da enfermagem ou dos técnicos de diagnóstico e terapêutica pode interferir com as estimativas de necessidades de médicos, sobretudo em períodos de fortes constrangimentos económicos e financeiros.

A caracterização demográfica dos médicos em Portugal revela uma classe profissional envelhecida, em que um terço dos seus efetivos em idade ativa atingirá os 70 anos até 2025. Este facto é importante na modelação de cenários de futuro, implicando a necessidade de renovação dos profissionais.

Por outro lado, devemos ter em atenção que qualquer alteração introduzida na capacidade de formação apenas produzirá efeitos a partir de 2020, uma vez que o sistema está já, em grande medida, condicionado pelo número de estudantes de Medicina em formação.

Tem, ainda, que se ter em linha de conta que a introdução, a curto-prazo, de medidas restritivas como, por exemplo, a limitação do acesso à formação pós-graduada, terá como consequência a criação de um contingente de diplomados em Medicina que, à luz das regras atualmente em vigor, não terão competência para o exercício autónomo da profissão (No âmbito do desenvolvimento do Estudo estimámos que a concretização do Cenário Com Limitações da Oferta Formativa traduzir-se-ia, em 2025, na existência de um contingente de 5450 diplomados em Medicina que não teriam acesso à formação específica do internato médico).

\section{CONCLUSÕES}

A determinação do número adequado de profissionais de saúde, nas suas diversas categorias profissionais e especializações, no momento certo e no local adequado, constitui um dos grandes desafios do planeamento e da gestão dos recursos humanos em saúde. Mas este é um exercício pleno de dificuldades, desde logo porque os determinantes de uma adequada definição do número e da tipologia de profissionais a afetar a determinado sistema de saúde são função de um conjunto numeroso de variáveis - grau de universalidade e cobertura do sistema de saúde, organização e níveis de serviço, políticas de saúde, sistemas de organização e gestão das estruturas de saúde, evolução demográfica e epidemiológica, evolução do conhecimento científico, das tecnologias de informação e das tecnologias de saúde, competências dos profissionais e respetivos conteúdos funcionais, etc.

No caso específico dos médicos, os tempos longos associados à formação destes profissionais (em Portugal, a formação específica de um médico tem uma duração mínima de 11 anos), introduz um elemento adicional de complexidade ao exercício de planeamento, exigindo uma anteci- 
pação e uma capacidade de previsão que comportam um risco elevado de desadequação à realidade.

Os trabalhos de investigação mais recentes sobre os determinantes da demografia dos profissionais de saú$\mathrm{de}^{2}$, concretamente no caso dos médicos, concluem que a tendência de aumento do pessoal médico tem sido uma constante ao longo das últimas três décadas. Estes trabaIhos perspetivam que esta tendência persistirá no futuro, em consequência da pressão crescente sobre o aumento dos cuidados de saúde a prestar às populações, tanto em quantidade como em qualidade, sendo esta tendência de aumento de profissionais apenas limitada pela capacidade das economias nacionais em sustentá-la. ${ }^{6}$

As conclusões do estudo ${ }^{11}$ que desenvolvemos apontam para a existência de um sistema de formação de médicos (pré e pós-graduada) com capacidade instalada para suprir as necessidades dos cenários modelados. Ou seja, da confrontação da capacidade de produção de médicos instalada no sistema, através da formação pré e pós-graduada, com os Cenários de Necessidades desenvolvidos, resulta a conclusão de que o stock de novos especialistas formados é suficiente para suprir as necessidades em todos eles, verificando-se mesmo a existência de especialistas que poderão ser excedentários relativamente às necessidades assistenciais do país.

Resulta, assim, a conclusão de que se verifica um de-

\section{REFERÊNCIAS}

1. Diallo K, Zurn P, Gupta N, Dal Poz M. Monitoring and evaluation of human resources for health: an international perspective. Hum Resour Health. 2003;1:3.

2. Danon-Hersch N, Paccaud F. Future Trends in Human Resources for Health Care: A Scenario Analysis; 2005. [Consultado 2014 Jan 16]. Disponivel em: http://books.google.pt/books/about/Future_Trends_in_Human_Resources_for_Hea.html?id=_W05QwAACAAJ\&pgis=1.

3. Santana P, Vaz A. Planeamento e gestão dos recursos humanos. In: Campos L, Borges M, Portugal R, editores. Governação dos Hospitais. Lisboa: Casa das Letras; 2009.

4. Chen L, Evans T, Anand S, Boufford JI, Brown H, M Chowdhury, et al. Human resources for health: overcoming the crisis. Lancet. 2004;364:1984-90.

5. Dussault G, Dubois CA. Human resources for health policies: a critical component in health policies. Hum Resour Health. 2003;1:1.

6. Development $\mathrm{O}$ for EC. The looming crisis in the health workforce: how can OECD countries respond? World Health Organization; 2008:99. [Consultado 2014 Jan 16]. Disponível em: http://www.who.int/workforcealliance/knowledge/resources/oecd_loomingcrisis/en/.

7. Bloor K, Maynard A. Planning human resources in health care: towards an economic approach, an international comparative review.; 2003:34. [Consultado 2014 Jan 16]. Available at: http://www.hrhresourcecenter. org/node/274.

8. Frenk J, Chen L, Bhutta ZA, Crisp N, Evans T, Fineberg H, et al. Health professionals for a new century: transforming education to strengthen health systems in an interdependent world. Lancet. 2010;376:1923-58. sajustamento entre o potencial de produção de médicos especialistas instalado e as respetivas necessidades modeladas. A dimensão estimada desse desajustamento varia entre os 8802 e os 3978 especialistas que, dependendo dos cenários, não seriam absorvidos pelo sistema de saúde.

A análise global desenvolvida não reflete, no entanto, a realidade de todas as especialidades da mesma forma. A análise da relação entre a capacidade produtiva instalada no sistema de formação nacional expressa no Modelo da Oferta, e as necessidades estimadas nos cenários que integram o Modelo das Necessidades, aplicada às 47 especialidades médicas reconhecidas pela Ordem dos Médicos, revela como principal conclusão (que deve ser mitigada pelo grau de incerteza que decorre das limitações informacionais e metodológicas referidas), que se verifica um excedente de capacidade de formação instalada em algumas das especialidades médicas e um défice na mesma capacidade formativa noutras especialidades.

\section{CONFLITOS DE INTERESSE}

Os autores declaram a inexistência de conflitos de interesse na realização do presente trabalho.

\section{FONTES DE FINANCIAMENTO}

Não existiram fontes externas de financiamento.

9. Santana P, Couceiro L, Alves I, Nogueira H, Costa C, Santos R. Caracterização demográfica dos profissionais de saúde no Sistema Nacional de Saúde Português. Lisboa: ACSS; 2007.

10. Santana P, Peixoto H, Loureiro A, Costa C, Nunes C, Duarte N. Estudo das necessidades previsionais de recursos humanos em saúde: médicos. Lisboa: ACSS; 2009.

11. Santana P, Peixoto H, Loureiro A, Costa C, Nunes C, Duarte N. Estudo de evolução prospectiva de médicos no Sistema Nacional de Saúde. Lisboa: Ordem dos Médicos; 2013.

12. Ono T, Lafortune G, Schoenstein M. Health workforce planning in OECD Countries: a review of 26 projection models from 18 countries. 2013 OECD Health Working Papers, $N^{\circ} 62$, OECD Publishing [Consultado em 2014 Jan 16]. Disponível em: http://dx.doi.org/10.1787/5k44t7872cwben.

13. Pérez PB, López-Valcárcel BG. Oferta y necesidad de especialistas médicos en España (2008-2025). Canarias: Universidad de las Palmas de Gran Canaria; 2009.

14. Roberfroid D, Stordeur S, Camberlin C, Voorde C Van de, Vrijens F, Léonard C. L'offre de médecins en Belgique. Situation actuelle et défis KCE reports 72B. Brussels; 2008.

15. Services USD of $H$ and $H$. The physician workforce: projections and research into current issues affecting supply and demand. USA: HRSA 2008. p.106.

16. Revisão do Regime de Internato Médico - Relatório Final. Lisboa: Secretário de Estado da Saúde, Governo de Portugal; 2012. 


\section{Demografia Médica em Portugal: Análise Prospetiva \\ Acta Med Port 2014:27:246-251}

Publicado pela Acta Médica Portuguesa, a Revista Científica da Ordem dos Médicos

Av. Almirante Gago Coutinho, 151

1749-084 Lisboa, Portugal.

Tel: +351 218428215

E-mail: submissao@actamedicaportuguesa.com

www.actamedicaportuguesa.com

ISSN:0870-399X | e-ISSN: 1646-0758

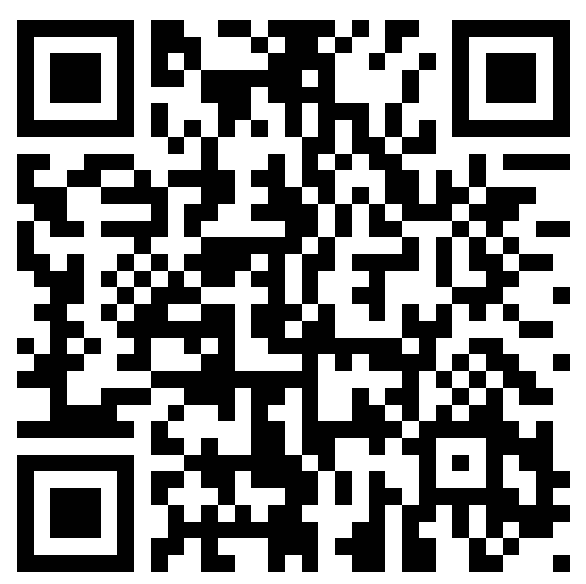

